\title{
The effect of a moderate intensity exercise programme on thymic cell function in undernourished rats
}

\author{
W. D. S. Cunha ${ }^{1}$, A. S. Yamashita ${ }^{1}$, M. V. Giampietro ${ }^{1}$, D. F. Souza ${ }^{1}$, A. S. Fernandes ${ }^{1}$, M. Vaisberg ${ }^{2}$, \\ W. Savino ${ }^{3}$, L. F. B. P. Costa Rosa ${ }^{1}$ and M. C. L. Seelaender ${ }^{1}$ \\ ${ }^{1}$ Institute of Biomedical Sciences, University of São Paulo, Brazil, ${ }^{2}$ São Paulo Medical School, UNIFESP, Brazil and \\ ${ }^{3}$ Oswaldo Cruz Institute, Fiocruz, Brazil
}

\begin{abstract}
Undernourishment is still a noticeable cause of impaired immunocompetence, and has been shown to be an important causal factor in the increased susceptibility to infectious diseases. Exercise modulates both innate and acquired immune response. The direction and magnitude of the changes, however, are influenced by numerous factors comprising the type, duration and intensity of exercise, as well as the conditioning level and age of the subject. Herein we have presently the effect of a moderate intensity exercise programme on immune cell function in undernourished rats. Rats $(n=40)$ were submitted to caloric restriction $(\mathrm{CR}, 50 \%)$ for 4 weeks, and then trained for 10 weeks at $60 \%$ VO2max, in a treadmill, 5 days a week, $1 \mathrm{~h}$ a day.

CR led to a reduction in thymus cellularity (48 and $59 \%$, respectively).

$\mathrm{CR}$ led to a reduction in concanavalin A-induced mitogenic response in lymphocyte from mesenteric lymph nodes (LFN) and spleen (48 and $59 \%$, respectively).

The moderate intensity training protocol restored thymic cellularity.

Additionally, the exercise protocol promoted a decrease in plasma corticosterone and an increase in plasma leptin concentration; both changes being strongly associated with the thymic recovery.

The present data reinforce that undernutrition decreases in vitro immune cell function and that a moderate intensity training protocol restores the thymic cellularity and lymphocyte mitogenic response.
\end{abstract}

This work was financially support by FAPESP 01/06655-1; Fiocruz, CNPq (Brazil). 